\title{
BI-UNITARY PERFECT NUMBERS
}

\author{
CHARLES R. WALL
}

\begin{abstract}
Let $d$ be a divisor of a positive integer $n$. Then $d$ is a unitary divisor if $d$ and $n / d$ are relatively prime, and $d$ is a bi-kmitary civisor if the greatest common unitary divisor of $d$ and $n / d$ is 1 . An integer is bi-unitaty perfect if it equals the sum of its proper biunitary divisors. The purpose of this paper is to show that there are only three bi-unitary perfect numbers, namely 6,60 and 90 .
\end{abstract}

A divisor $d$ of an integer $n$ is a unitary divisor if $d$ and $n / d$ are relatively prime. A divisor $d$ of an integer $n$ is a bi-unitary clivisor if the greatest common unitary divisor of $d$ and $n / d$ is 1 . Let $\sigma(n)$ be the sum of the divisors of $n$, let $\sigma^{*}(n)$ be the sum of the unitary divisors of $n$, and let $\sigma^{* *}(n)$ be the sum of the bi-unitary divisors of $n$.

We say that $N$ is unitary perfect if $\sigma^{*}(N)=2 N$. Subbarao and Warren [2] showed that $6,60,90$ and 87360 are the first four unitary perfect numbers; Wall reported [3] that

$$
146,361,946,186,458,562,560,000=2^{18} 3 \cdot 5^{4} 7 \cdot 11 \cdot 13 \cdot 19 \cdot 37 \cdot 79 \cdot 109 \cdot 157 \cdot 313
$$

is also unitary perfect and later showed [4] it to be the next such number after 87360 . Subbarao [1] has conjectured that there are only finitely many unitary perfect numbers.

We say that $N$ is bi-unitary perfect if $\sigma^{* *}(N)=2 N$. The purpose of this paper is to show that the first three unitary perfect numbers, i.e., 6,60 and 90 , are the only bi-unitary perfect numbers.

One easily verifies that $\sigma^{* *}$ is multiplicative and that if $p$ is prime and $e \geqq 1$, then

if $\epsilon$ is odd, and

$$
\sigma^{* *}\left(p^{e}\right)=\sigma\left(p^{e}\right)=\left(p^{e+1}-1\right) /(p-1)
$$

$$
\sigma^{* *}\left(p^{e}\right)=\left(p^{e+1}-1\right) /(p-1)-p^{e / 2}
$$

if $e$ is even. Hence $\sigma^{* *}(n) \leqq \sigma(n)$ with equality if and only if every prime which divides $n$ does so an odd number of times. It also follows immediately that $\sigma^{* *}(n)$ is odd if and only if $n$ is 1 or a power of 2 ; consequently, each odd prime power unitary divisor of $n$ contributes at least one factor 2 to $\sigma^{* *}(n)$.

Received by the editors June 10, 1971.

AMS 1970 subject classifications. Primary 10A20; Secondary 10A99.

Key words and phrases. Bi-unitary divisors, perfect numbers. 
Let $\phi$ be Euler's totient.

THEOREM 1. There are no odd bi-unitary perfect numbers.

Proof. If $\sigma^{* *}(N)=2 N$ and $N$ is odd, then $N$ must be a prime power, say $N=p^{e}$. But then

$$
\sigma^{* *}\left(p^{e}\right) / p^{e} \leqq \sigma\left(p^{e}\right) / p^{e}<p^{e} / \phi\left(p^{e}\right)=p /(p-1) \leqq \frac{3}{2},
$$

so $N$ cannot be bi-unitary perfect.

We define $f(n)=\sigma^{* *}(n) / n$ and note that if $p$ is any prime, then

and

$$
1=f(1)<f\left(p^{2}\right)<f(p)<f\left(p^{4}\right)<f\left(p^{3}\right),
$$

$$
f\left(p^{\alpha}\right)<f\left(p^{\alpha+2}\right)<p /(p-1)
$$

for all natural numbers $\alpha$, and $f\left(p^{\alpha}\right)>f\left(p^{\alpha+1}\right)$ if $\alpha$ is odd. Also, we remark that $f(N)=2$ if and only if $N$ is bi-unitary perfect.

THEOREM 2. The only even bi-unitary perfect numbers are 6,60 and 90 .

Proof. Henceforth we assume that $N$ is bi-unitary perfect and even, and write $N=2^{a} M=2^{a} X Y$ where $X=\sigma^{* *}\left(2^{a}\right)$. Our scheme of proof is to establish that: (i) if $a$ is odd, then $a=1$; (ii) if $a=1$, then $N=6$ or $N=90$; (iii) if $a=2$, then $N=60$; (iv) if $a \geqq 6$, then $(N, 3)=1$; (v) $a=4$ and $a=6$ are impossible; and (vi) $a \geqq 8$ is impossible.

If $a$ is odd, then $3 \mid X$. If $a \geqq 3$, then $f\left(2^{a}\right) \geqq \frac{15}{8}$, so

$$
f(N) \geqq f(8) f(9)=\left(\frac{15}{8}\right)\left(\frac{1}{9}\right)=\frac{25}{12}>2,
$$

contradicting the assumption that $f(N)=2$. Thus (i) is proved.

If $a=1$, then $3 \mid N$ and $N$ has at most two distinct odd prime divisors. If $3^{3} \mid N$, then

$$
f(N) \geqq\left(\frac{3}{2}\right)\left(\begin{array}{cc}
1 & 1 \\
8 & 1
\end{array}\right)>2 .
$$

If $3 \| N$, then $N=6$. If $3^{2} \| N$, then $5 \mid N$ as $1+3^{2}=2 \cdot 5$ and 5 cannot divide $N$ exactly twice without $N$ having three distinct odd prime divisors. Then $f(N)>2$ unless $5 \| N$, which yields $N=90$. Thus (ii) is proved.

If $a=2$, then $5 \mid N$ and $N$ has at most three distinct odd prime divisors. Suppose $3 \nmid N$, and let $N=4 M$ with $M$ odd. Then since

and

$$
f(N)<5 M / 4 \phi(M)
$$

$$
\begin{aligned}
(5 \cdot 7 \cdot 11 \cdot 13) /(4 \cdot 6 \cdot 10 \cdot 12) & <(5 \cdot 5 \cdot 11 \cdot 13) /(4 \cdot 4 \cdot 10 \cdot 12) \\
& <(5 \cdot 5 \cdot 7 \cdot 13) /(4 \cdot 4 \cdot 6 \cdot 12)<2,
\end{aligned}
$$

we must have $N=2^{2} 5^{b} 7^{c} 11^{d}$ for some choice of positive exponents $b, c$ and $d$. Moreover, $A=\sigma^{* *}\left(11^{d}\right)$ must be divisible by 2 exactly once and 
not by 3 , which requires that $d$ be even, say $d=2 e$. Then

$$
A=\left(1+1 \mathrm{i}^{e+1}\right)\left(1 !^{e}-1\right) / 10
$$

and 5 and 7 are the only odd primes which can divide A. From congruences modulo 7 , it is clear that if $7 \mid A$ then $3 \mid e$; but then the factor $\left(11^{e}-1\right)$ is a multiple of 19 , so $19 \mid A$, a contradiction. Now, $A$ cannot be a power of 2 , because to avoid having $1+11^{e+1}$ be a multiple of 3 , we must have $e$ odd, whence $\left(11^{e}-1\right) / 10$ is odd. If $7 \nmid A$, then $5 \mid A$, so $5^{2} \mid\left(11^{e}-1\right)$ and consequently $5 \mid e$; but then $3221 \mid A$, a contradiction. Thus $3 \mid N$.

If $a=2$ and $3 \mid N$ we write $N=2^{2} 3^{b} 5^{c} N^{\prime}$ where $\left(N^{\prime}, 30\right)=1$; in fact, $N^{\prime}$ is either 1 or a prime power. If neither $b$ nor $c$ is 2 , then

$$
f(N) \geqq\left(\frac{5}{4}\right)\left(\frac{4}{3}\right)\left(\frac{8}{5}\right)=2
$$

with equality only for $N=60$. If $c=2$ then $13 \mid N^{\prime}$, and 13 cannot divide $N$ exactly twice or else $17 \mid N$ and $N$ has at least four odd prime divisors. If $b \geqq 5$ then

$$
f(N) \geqq\left(\frac{5}{4}\right)\left(\frac{1068}{729}\right)\left(\frac{26}{2} \frac{6}{5}\right)\left(\frac{14}{13}\right)>2 ;
$$

if $b$ is 1 or 3 there are too many factors 2 in the numerator of $f(N)$, and if $b=4$, there is an excess factor 7 ; if $b=2$, then

$$
f(N) \leqq\left(\frac{5}{4}\right)\left(\frac{10}{9}\right)\left(\frac{2}{2} \frac{6}{5}\right)\left(\frac{13}{1} \frac{3}{2}\right)<2 .
$$

Thus we cannot have $c=2$. If $b=2$, then as $5^{2} \mid \sigma^{* *}\left(2^{2} 3^{2}\right)$ we must have $c \geqq 2$. So $c \geqq 3$ as the case $b=c=2$ has already been eliminated. If $7 \nmid N$ then

$$
f(N)<\left(\frac{5}{4}\right)\left(\frac{10}{9}\right)\left(\frac{5}{4}\right)\left(\frac{1}{1} \frac{1}{0}\right)<2 .
$$

Thus, $7 \mid N$. If $c=4$, then $N$ has too many factors 3 . The possibilities $7\left\|N, 7^{3}\right\| N$ and $7^{4} \| N$ lead to too many factors 2 in $N$, while $7^{2} \| N$ implies that $f(N)<2$. Therefore, $7^{5} \mid N$ and if $c=3$ or $c \geqq 5$, then

$$
f(N) \geqq\left(\frac{5}{4}\right)\left(\frac{10}{4}\right) f\left(5^{6} 7^{6}\right)>2 .
$$

Hence (iii) is proved.

If $N$ is divisible by $3 \cdot 2^{6}$ then

$$
f(N) \geqq\left(\frac{119}{64}\right)\left(\frac{19}{9}\right)>2,
$$

contradicting the fact that $f(N)=2$. Thus (iv) is proved.

If $a=4$, then $N=16 \cdot 27 N^{\prime}$ with $N^{\prime}$ odd. Then

$$
f(N) \geqq\left(\frac{2}{16}\right)\left(\frac{11}{81} \frac{2}{1}\right)>2,
$$

a contradiction. If $a=6$, then $7 \cdot 17 \mid M$. If 7 does not divide $M$ exactly twice, then $f(N)>2$; thus $7^{2} \| M$, so $5^{2} \mid M$. If $5^{3} \mid M$ then $f(N)>2$. Hence 
$5^{2} \| M$, so $13 \mid M$ and 13 does not divide $M$ exactly twice or else $5^{3} \mid M$. Then

$$
f(N) \geqq\left(\frac{119}{64}\right)\left(\frac{50}{49}\right)\left(\frac{2}{2} \frac{6}{5}\right)\left(\frac{1}{1} \frac{4}{3}\right)\left(\frac{29}{2} \frac{0}{8}\right)>2,
$$

a contradiction which establishes $(\mathrm{v})$.

If $a \geqq 8$, then $(N, 5)=1$ or else $f(N) \geqq\left(\frac{49}{2} \frac{95}{6}\right)\left(\frac{2}{2} \frac{6}{5}\right)>2$, a contradiction. We set $a=2 b$ with $b \geqq 4$. Then

$$
X=\sigma^{* *}\left(2^{a}\right)=2^{2 b+1}-2^{b}-1=\left(2^{b}-1\right)\left(1+2^{b+1}\right)
$$

is composite. Since $(3, M)=1$ and $X \mid M$, and $1+2^{b+1}=2\left(2^{b}-1\right)+3$, we know the factors $2^{b}-1$ and $1+2^{b+1}$ are relatively prime.

Let $p$ be any prime dividing $X$, and suppose $p^{c} \| M$. If $c \neq 2$, then

$$
1+p^{-1} \leqq f\left(p^{c}\right) \leqq f(M)=2^{2 b+1} /\left(2^{2 b+1}-2^{b}-1\right),
$$

which requires that $p \geqq 2^{b+1}-3+2 /\left(1+2^{b}\right)>X^{1 / 2}$, whence $p=X$, contradicting the fact that $X$ is composite. Thus any prime that divides $X$ must divide $M$ exactly twice. Then

$$
1+p^{-2}=f\left(p^{2}\right) \leqq f(M)
$$

requires that $p^{4}>X$. Hence $X$ has no more than three distinct prime factors, and $(X, 30)=1$. But $X=\left(2^{b}-1\right)\left(1+2^{b+1}\right)$, so one of the factors must be prime. As $(X, 3)=1$, we must have $b$ odd. With the restriction that $b$ be odd, either

or

$$
2^{b}-1 \equiv 1(\bmod 10) \text { and } 1+2^{b+1} \equiv 5(\bmod 10)
$$

$$
2^{b}-1 \equiv 1+2^{b+1} \equiv 7(\bmod 10) .
$$

The first case is eliminated as $(N, 5)=1$. In the second case, one of the two numbers must be prime: call this prime $p$. Then $p \equiv 7(\bmod 10)$ and $p^{2} \| N$. But $\sigma^{* *}\left(p^{2}\right)=1+p^{2}$ is then a multiple of 5 , so $5 \mid N$, a contradiction.

Hence the theorem is proved.

The author thanks the referee for providing a portion of part (iii) of the proof of Theorem 2 .

\section{REFERENCES}

1. M. V. Subbarao, Are there an infinity of unitary perfect numbers?, Amer. Math. Monthly 77 (1970), 389-390.

2. M. V. Subbarao and L. J. Warren, Unitary perfect numbers, Canad. Math. Bull. 9 (1966), 147-153. MR 33 \#3994.

3. C. R. Wall, A new unitary perfect number, Notices Amer. Math. Soc. 16 (1969), 825. Abstract \#69T-A139.

4. - The fifth unitary perfect number, Canad. Math. Bull. (to appear).

Department of Mathematics, East Texas State University, Commerce, Texas 75428 